Draft version June 21, 2021

Typeset using $\mathrm{LAT}_{\mathrm{E}} \mathrm{X}$ twocolumn style in AASTeX61

submission

\title{
MOLECULAR GAS IN DEBRIS DISKS AROUND YOUNG A-TYPE STARS
}

\author{
Attila Moór,${ }^{1}$ Michel Curé, ${ }^{2}$ Ágnes Kóspál, ${ }^{1,3}$ Péter Ábrahám, ${ }^{1}$ Timea Csengeri, ${ }^{4}$ Carlos Eiroa, ${ }^{5}$ \\ Diah Gunawan, ${ }^{2}$ Thomas Henning, ${ }^{3}$ A. Meredith Hughes, ${ }^{6}$ Attilla Juhász, ${ }^{7}$ Nicole Pawellek, ${ }^{3}$ And \\ MARK WYATT $^{7}$ \\ ${ }^{1}$ Konkoly Observatory, Research Centre for Astronomy and Earth Sciences, Hungarian Academy of Sciences, Konkoly-Thege Miklós út \\ 15-17, 1121 Budapest, Hungary \\ ${ }^{2}$ Instituto de Física y Astronomía, Universidad de Valparaíso, Valparaíso, Chile \\ ${ }^{3}$ Max Planck Institute for Astronomy, Königstuhl 17, D-69117 Heidelberg, Germany \\ ${ }^{4}$ Max Planck Institute for Radio Astronomy, Auf dem Hügel 69, 53121 Bonn, Germany \\ ${ }^{5}$ Dpto. Física Teórica, Universidad Autónoma de Madrid, Cantoblanco, 28049, Madrid, Spain \\ ${ }^{6}$ Department of Astronomy, Van Vleck Observatory, Wesleyan University, 96 Foss Hill Drive, Middletown, CT 06459, USA \\ ${ }^{7}$ Institute of Astronomy, University of Cambridge, Madingley Road, Cambridge CB3 OHA, UK
}

(Received date; Revised date; Accepted date)

Submitted to ApJL

\begin{abstract}
According to the current paradigm of circumstellar disk evolution, gas-rich primordial disks evolve into gas-poor debris disks composed of second-generation dust. To explore the transition between these phases, we searched for ${ }^{12} \mathrm{CO},{ }^{13} \mathrm{CO}$, and $\mathrm{C}^{18} \mathrm{O}$ emission in seven dust-rich debris disks around young A-type stars, using ALMA in Band 6 . We discovered molecular gas in three debris disks. In all these disks, the ${ }^{12} \mathrm{CO}$ line was optically thick, highlighting the importance of less abundant molecules in reliable mass estimates. Supplementing our target list by literature data, we compiled a volume-limited sample of dust-rich debris disks around young A-type stars within $150 \mathrm{pc}$. We obtained a $\mathrm{CO}$ detection rate of $11 / 16$ above a ${ }^{12} \mathrm{CO} \mathrm{J}=2-1$ line luminosity threshold of $\sim 1.4 \times 10^{4} \mathrm{Jy} \mathrm{km} \mathrm{s}^{-1} \mathrm{pc}^{2}$ in the sample. This high incidence implies that the presence of $\mathrm{CO}$ gas in bright debris disks around young A-type stars is likely more the rule than the exception. Interestingly, dust-rich debris disks around young FG-type stars exhibit, with the same detectability threshold as for A-type stars, significantly lower gas incidence. While the transition from protoplanetary to debris phase is associated with a drop of dust content, our results exhibit a large spread in the CO mass in our debris sample, with peak values comparable to those in protoplanetary Herbig Ae disks. In the particularly CO-rich debris systems the gas may have primordial origin, characteristic of a hybrid disk.
\end{abstract}

Keywords: circumstellar matter — infrared: stars — stars: individual (HD 121191, HD 121617, HD 131488) 


\section{INTRODUCTION}

During their early evolution, newborn stars are surrounded by massive gas-rich circumstellar disks. Most these primordial disks dissipate by the age of $10 \mathrm{Myr}$ (e.g., Alexander et al. 2014), partly because their material is incorporated to planetesimals and planets. Later, the collisional erosion of the planetesimals may produce fresh dust (Wyatt 2008). The tenuous debris disk, formed by these second-generation dust particles, may accompany the star almost throughout its life. The transformation from primordial to debris disk is perhaps the most radical change during a disk's lifetime, whose details are still little understood (Wyatt et al. 2015). The evolution of the dust component is relatively well known, because infrared and submillimeter continuum observations outline how the dust mass decreases with time (Wyatt 2008). Due to the rarity of gas detections in debris disks, we know significantly less about the gas component. Mature debris disks are expected to have low gas-to-dust ratio, because processes like collisions, sublimation, and photodesorption from icy grains or planetesimals can produce only a small amount of secondary gas and moreover the most detectable species like CO photodissociate rapidly in the interstellar radiation field (Matthews et al. 2014).

Recent surveys with single dish radio telescopes and the ALMA interferometer identified a growing population of debris disks containing detectable amounts of molecular gas. Detecting rotational transitions of $\mathrm{CO}$ molecules, up to now twelve CO-bearing debris disks have been discovered: 49 Cet (Zuckerman et al. 1995), HD 21997 (Moór et al. 2011), $\beta$ Pic (Dent et al. 2014), HD 131835 (Moór et al. 2015b), HD 181327 (Marino et al. 2016), HD 110058, HD 138813, HD 146897, HD 156623 (Lieman-Sifry et al. 2016), HD 32297 (Greaves 2016), $\quad \eta$ Crv (Marino et al. 2017), and Fomalhaut (Matrà et al. 2017b). These disks share several distinctive physical characteristics: most of them are young (<50 Myr), surround A-type stars, exhibit high fractional luminosity, and have a dust component that is relatively cold $(<140 \mathrm{~K})$, resembling the Kuiper belt rather than the asteroid belt in Solar System terminology. The gas component may have a secondary origin, being produced from icy material within the planetesimal belt.However, considering the youth of the systems, it may also be the remnant of the protoplanetary disk (primordial origin). There is now proof for secondary gas in four systems: $\beta$ Pic, (Matrà et al. 2017a), Fomalhaut (Matrà et al. 2017b), HD 181327 (Marino et al. 2016), and $\eta$ Crv (Marino et al. 2017). The large amount of gas in HD 21997 and HD 131835 is difficult to explain within the framework of current secondary gas production models (Kóspál et al. 2013; Kral et al. 2017), raising the possibility that the gas is primordial while the dust is secondary, forming a hybrid disk (Kóspál et al. 2013, Moór et al. in prep.).

We have only a few constraints on how circumstellar disks reach the gas-poor phase, how closely the gas evolution is coupled to the disappearance of the primordial dust, when the secondary gas production starts, and how long the disk can retain the primordial gas. Motivated by these open questions and to explore the conditions under which a disk could keep its primordial gas component longer, we initiated a systematic investigation of molecular gas in debris disks. We focus on young A-type stars, and determine the incidence and physical parameters of their molecular gas component. Here we report on new ALMA observations of seven disks, which were selected to complete a volume limited sample of dust-rich debris disks around young A-type stars within 150 pc. We targeted all three main CO isotopologues, since ${ }^{12} \mathrm{CO}$ alone does not provide reliable gas mass estimates if optically thick.

\section{SAMPLE SELECTION}

In order to obtain a complete census of all debris systems within 150 pc which are similar to the known CObearing debris disks, we adopted the following criteria: (1) A-type host star; (2) $5 \times 10^{-4}<L_{\text {disk }} / L_{\text {bol }}<10^{-2}$, thus the fractional luminosity is higher than the lowest value (in HD 21997) found in any CO-bearing debris disk younger than $50 \mathrm{Myr}$, but lower than typical values in protoplanetary disks; (3) dust temperature <140 K, to ensure that - similarly to all previous detections - the disks have a cold dust component; (4) $\geq 70 \mu \mathrm{m}$ detection with Spitzer/MIPS or Herschel/PACS; (5) alage between 10 and $50 \mathrm{Myr}$. We searched the literature (Ballering et al. 2013; Chen et al. 2014; Melis et al. 2013; Moór et al. 2011) and found 17 systems within $150 \mathrm{pc}$ which satisfied these criteria. The age estimates for all systems are well established, being based on membership in young moving groups or associations, except one target, HD 32297. We excluded from this sample those 10 systems where sensitive ALMA observations were already available. The remaining seven disks formed the target list of our dedicated ALMA survey. Remarkably, all these objects belong to the Sco-Cen association. Table 1 gives the main parameters for all 17 sources, including our present targets.

\section{OBSERVATIONS}

Our targets were observed with ALMA in Band 6 (project 2015.1.01243.S, PI: M. Curé). Table 2 shows the $\log$ of observations. We used identical correlator 
Table 1. Stellar and disk parameters

\begin{tabular}{|c|c|c|c|c|c|c|c|c|c|c|c|}
\hline Name & Spt. & $\begin{array}{l}\text { Dist. } \\
\text { (pc) } \\
(3) \\
\end{array}$ & $\begin{array}{l}\text { Lum. } \\
\left(L_{*}\right) \\
(4) \\
\end{array}$ & (5) & $\begin{array}{c}\text { Age } \\
(\mathrm{Myr}) \\
(6) \\
\end{array}$ & $\begin{array}{l}T_{\text {dust }} \\
(\mathrm{K}) \\
(7) \\
\end{array}$ & $L_{\mathrm{disk}} / L_{\mathrm{bol}}$ & $\begin{array}{c}M_{\text {dust }} \\
\left(M_{\oplus}\right) \\
(9) \\
\end{array}$ & (10) & $\begin{array}{c}S_{12} \mathrm{CO} \\
\left(\mathrm{Jy} \mathrm{km} \mathrm{s}^{-1}\right) \\
(11) \\
\end{array}$ & $\begin{array}{l}M_{\mathrm{CO}} \\
\left(M_{\oplus}\right) \\
(12) \\
\end{array}$ \\
\hline \multicolumn{12}{|c|}{ Current sample } \\
\hline HD 98363 & $\mathrm{~A} 2 \mathrm{~V}$ & 123.6 & 10.5 & $\operatorname{LCC}(7)$ & $15(4)$ & $295 / 112$ & $9.2 \times 10^{-4}(2)$ & $6.8 \times 10^{-3}(8)$ & $\mathrm{N}$ & $<0.036(8)$ & $\cdots$ \\
\hline HD 109832 & $\mathrm{~A} 9 \mathrm{~V}$ & 111.9 & 5.3 & $\mathrm{LCC}(7)$ & $15(4)$ & $186 / 92$ & $7.6 \times 10^{-4}(1)$ & $<6.7 \times 10^{-3}(8)$ & $\mathrm{N}$ & $<0.033(8)$ & $\ldots$ \\
\hline HD 121191 & $\mathrm{~A} 5 \mathrm{IV} / \mathrm{V}$ & 135.9 & 8.2 & LCC/UCL (4) & $15-16(4)$ & $555 / 118$ & $4.7 \times 10^{-3}(9)$ & $9.5 \times 10^{-3}(8)$ & $\mathrm{Y}$ & $0.23 \pm 0.04(8)$ & $2.7 \times 10^{-3}$ \\
\hline HD 121617 & $\mathrm{~A} 1 \mathrm{~V}$ & 128.2 & 17.0 & UCL (2) & $16(4)$ & 105 & $4.8 \times 10^{-3}(4)$ & $1.4 \times 10^{-1}(8)$ & $\mathrm{Y}$ & $1.27 \pm 0.13(8)$ & $1.8 \times 10^{-2}$ \\
\hline HD 131488 & $\mathrm{~A} 1 \mathrm{~V}$ & 147.7 & 13.1 & UCL (4) & $16(4)$ & $570 / 94$ & $5.5 \times 10^{-3}(9)$ & $3.2 \times 10^{-1}(8)$ & $\mathrm{Y}$ & $0.78 \pm 0.08(8)$ & $8.9 \times 10^{-2}$ \\
\hline HD 143675 & $\mathrm{~A} 5 \mathrm{IV} / \mathrm{V}$ & 113.4 & 7.1 & UCL (7) & $16(4)$ & $374 / 127$ & $5.9 \times 10^{-4}(2)$ & $<1.1 \times 10^{-2}(8)$ & $\mathrm{N}$ & $<0.078(8)$ & $\ldots$ \\
\hline HD 145880 & B9.5V & 127.9 & 32.8 & UCL (7) & $16(4)$ & $196 / 70$ & $1.1 \times 10^{-3}(1)$ & $<2.6 \times 10^{-2}(8)$ & $\mathrm{N}$ & $<0.071(8)$ & $\cdots$ \\
\hline
\end{tabular}

Other systems

\begin{tabular}{|c|c|c|c|c|c|c|c|c|c|c|c|}
\hline 49 Cet & $\mathrm{A} 1 \mathrm{~V}$ & 59.4 & 16.4 & ARG (8) & $40(5)$ & $155 / 56$ & $1.1 \times 10^{-3}(5)$ & $1.7 \times 10^{-1}(2)$ & $\mathrm{Y}$ & $2.00 \pm 0.30(3)$ & $\geq 1.9 \times 10^{-4}$ \\
\hline HD 21997 & $\mathrm{~A} 3 \mathrm{IV} / \mathrm{V}$ & 71.9 & 11.2 & COL (5) & $42(1)$ & 61 & $5.7 \times 10^{-4}(5)$ & $1.1 \times 10^{-1}(7)$ & $\mathrm{Y}$ & $2.17 \pm 0.23(4)$ & $6.0 \times 10^{-2}$ \\
\hline HD 32297 & A6V & 112.4 & 6.2 & - & $<30(2)$ & $292 / 88$ & $4.4 \times 10^{-3}(2)$ & $2.0 \times 10^{-1}(4)$ & Y & $5.11 \pm 0.49(1)$ & $\geq 1.5 \times 10^{-3}$ \\
\hline$\beta$ Pic & $\mathrm{A} 6 \mathrm{~V}$ & 19.4 & 8.7 & BPMG (1) & $23(3)$ & 85 & $2.6 \times 10^{-3}(3,7)$ & $4.2 \times 10^{-2}(1)$ & Y & $4.55 \pm 0.52(6)$ & $3.4 \times 10^{-5}$ \\
\hline HD 95086 & A8III & 90.4 & 7.0 & LCC (7) & $15(4)$ & $184 / 54$ & $1.7 \times 10^{-3}(5)$ & $2.2 \times 10^{-1}(6)$ & $\mathrm{N}$ & $<0.014(7)$ & $\ldots$ \\
\hline HR 4796 & $\mathrm{~A} 0 \mathrm{~V}$ & 72.8 & 23.4 & TWA (6) & $10(5)$ & 108 & $4.6 \times 10^{-3}(8)$ & $1.4 \times 10^{-1}(5)$ & $\mathrm{N}$ & $<6.15(2)$ & $\cdots$ \\
\hline HD 110058 & $\mathrm{~A} 0 \mathrm{~V}$ & 107.4 & 5.9 & LCC (7) & $15(4)$ & $499 / 112$ & $1.4 \times 10^{-3}(2)$ & $2.9 \times 10^{-2}(3)$ & $\mathrm{Y}$ & $0.09 \pm 0.02(5)$ & $\geq 2.5 \times 10^{-5}$ \\
\hline HD 131835 & A2IV & 122.7 & 9.2 & UCL (7) & $16(4)$ & $176 / 71$ & $3.0 \times 10^{-3}(6)$ & $2.5 \times 10^{-1}(3)$ & $\mathrm{Y}$ & $0.80 \pm 0.04(5)$ & $3.2 \times 10^{-2}$ \\
\hline HD 138813 & $\mathrm{~A} 0 \mathrm{~V}$ & 150.8 & 24.5 & US (7) & $10(4)$ & $194 / 94$ & $9.0 \times 10^{-4}(2)$ & $1.2 \times 10^{-1}(3)$ & $\mathrm{Y}$ & $1.41 \pm 0.08(5)$ & $\geq 7.5 \times 10^{-4}$ \\
\hline HD 156623 & $\mathrm{~A} 0 \mathrm{~V}$ & 118.3 & 14.8 & UCL (3) & $16(4)$ & $605 / 123$ & $5.0 \times 10^{-3}$ & $3.2 \times 10^{-2}(3)$ & $\mathrm{Y}$ & $1.18 \pm 0.04(5)$ & $\geq 3.9 \times 10^{-4}$ \\
\hline
\end{tabular}

Note- Column (1): Target name. Column (2): Spectral type (from SIMBAD, except for HD 32297, see Rodigas et al. 2014). Column (3): Distance (from Hipparcos parallax when available (van Leeuwen 2007), except for HD 121617 and HD 121191, see Lindegren et al. 2016, and for HD 131488, whose kinematic distance was derived based on its membership in UCL). Column (4): Luminosity. Column (5): Moving group membership. ARG: Argus moving group; BPMG: $\beta$ Pic moving group; COL: Columba moving group; LCC: Lower Centaurus Crux association; UCL: Upper Centaurus Lupus association; US: Upper Scorpius association. References for membership assignments: (1) Barrado y Navascués et al. (1999); (2) Hoogerwerf (2000); (3) Lieman-Sifry et al. (2016); (4) Melis et al. (2013); (5) Moór et al. (2006); (6) Webb et al. (1999); (7) de Zeeuw et al. (1999); (8) Zuckerman \& Song (2012). Column (6): Stellar age. References for age estimates (the age of the corresponding group in case of group members): (1) Bell et al. (2015); (2) Kalas (2005); (3) Mamajek \& Bell (2014); (4) Pecaut \& Mamajek (2016); (5) Torres et al. (2008). Column (7): Dust temperature. For references see Col. (8). Column (8): Fractional luminosity of the disk. References: (1) Ballering et al. (2013); (2) Chen et al. (2014); (3) Dent et al. (2014); (4) Moór et al. (2011); (5) Moór et al. (2015b); (6) Moór et al. (2015a); (7) Rhee et al. (2007); (8) Riviere-Marichalar et al. (2014); (9) Vican et al. (2016). For HD 156623, the characteristic dust temperatures and the fractional luminosity were derived by fitting a two-component modified blackbody to the excess SED. Column (9): Dust mass. References for the utilized (sub)millimeter observations: (1) Dent et al. (2014); (2) Hughes et al. (2017); (3) Lieman-Sifry et al. (2016); (4) Meeus et al. (2012); (5) Sheret et al. (2004); (6) Su et al. in prep.; (7) Williams \& Andrews (2006); (8) this work. Column (10): CO detection status. Column (11): Integrated line fluxes or 3 $\sigma$ upper limits for ${ }^{12} \mathrm{CO} J=2-1$ ( $J=3-2$ for HR 4796). References: (1) Greaves et al. (2016); (2) Hales et al. (2014); (3) Hughes et al. (2008); (4) Kóspál et al. (2013); (5) Lieman-Sifry et al. (2016); (6) Matrà et al. (2017a); (7) Su et al. in prep.; (8) this work. Column (12): Mass of CO gas (for details see Sect. 4).

setup in each case. Two spectral windows centered at 217 and $233.5 \mathrm{GHz}$ were dedicated to continuum measurements, providing $1875 \mathrm{MHz}$ bandwidth individually. The other two spectral windows were tuned to cover the ${ }^{12} \mathrm{CO},{ }^{13} \mathrm{CO}$, and $\mathrm{C}^{18} \mathrm{O} \mathrm{J}=2-1$ lines. Observations of the ${ }^{12} \mathrm{CO}(2-1)$ transition were performed using a window centered at $230.743 \mathrm{GHz}$ with a bandwidth of $468.75 \mathrm{MHz}$, while the isotopologue lines were measured together in a window centered at $219.492 \mathrm{GHz}$ with a bandwidth of $1875 \mathrm{MHz}$. The spectral resolutions in the two windows were $244 \mathrm{kHz}\left(0.32 \mathrm{kms}^{-1}\right)$ and $976 \mathrm{kHz}\left(1.33 \mathrm{kms}^{-1}\right)$, respectively.Calibration and imaging were done using the standard ALMA reduction tool Common Astronomy Software Applications (CASA v4.5; McMullin et al. 2007). To extract the continuum image and spectral cubes of different lines from the calibrated visibilities, we used the CASA task clean with Briggs weighting using a robustness parameter of 0.5 . For the line data, we first fitted a continuum to the linefree channels and subtracted it in the $u v$ space using the uvcontsub task. The continuum image was compiled by concatenating the dedicated continuum spectral 
windows and line free channels of the other two spectral windows. Beam sizes, position angles, and rms noises are also summarized in Table 2.

\section{RESULTS}

Continuum data.-Fig. 1 presents the $1.3 \mathrm{~mm}$ continuum images of our targets. For HD 121191, HD 121617 , HD 131488, and HD 98363, we detected significant (peak $>3 \sigma$ ) signal towards or close to the position of the central star. For HD 109832, the peak emission close to the stellar position is only $2.8 \sigma$. The remaining two objects were undetected. Continuum flux densities of HD 98363 and HD 121191 were determined by fitting a point source to their visibility data using the CASA uvmodelfit task. To derive the integrated flux density of the bright extended disks around HD 121617 and HD 131488 we used the uvmultifit task (Martí-Vidal et al. 2014) to fit a Gaussian ring model to the visibilities. These models also provide structural information on these disks (see Sect. 5). We determined upper limits for the nondetected sources by measuring the flux at random positions in the images in an aperture radius corresponding to $100 \mathrm{au}$. The measured fluxes and $3 \sigma$ upper limits are listed in Tab. 2, along with uncertainties containing $10 \%$ absolute calibration error added quadratically.

Assuming optically thin emission, the measured flux densities were converted to dust masses using

$$
M_{d}=\frac{F_{\nu} d^{2}}{B_{\nu}\left(T_{\text {dust }}\right) \kappa_{\nu}},
$$

where $d$ is the distance, $B_{\nu}\left(T_{\text {dust }}\right)$ is the Planck function at $1.3 \mathrm{~mm}$ for a characteristic dust temperature $T_{\text {dust }}$ taken from Tab. 1 , while $\kappa_{\nu}$ is the opacity at $1.3 \mathrm{~mm}$ for which a value of $2.3 \mathrm{~cm}^{2} \mathrm{~g}^{-1}$ was adopted (Andrews et al. 2013).

For consistency, the dust masses of the other ten disks from Table 1 were also computed using submillimeter/millimeter data collected from the literature and adopting an opacity value of $\kappa_{\nu}=$ $2.3\left(\frac{\nu}{230 \mathrm{GHz}}\right)^{0.7} \mathrm{~cm}^{2} \mathrm{~g}^{-1}$. Taking into account uncertainties in measured flux densities, distances, and dust temperature values, the formal uncertainty of our dust mass estimates are between 11 and 30\%. However, there are other factors that can influence the estimates. The emitting dust grains were characterized by a single temperature, that was derived from the analysis of the SED and therefore reflects the temperature of the smallest grains and not of those large ones that we observe at millimeter wavelengths. The latter grains are probably colder, thus, by using the SED-based $T_{\text {dust }}$, we may underestimate the dust mass. Even more serious uncertainty can be related to the adopted $\kappa_{\nu}$ value.
Depending on the chemical compositions, shape, and size distribution of dust grains, the millimeter opacity can vary significantly (Miyake \& Nakagawa 1993; Ossenkopf \& Henning 1994), thereby introducing an uncertainty of a factor of several in the calculation. The obtained dust masses are listed in Table 1.

Line data. - We detected significant $\mathrm{CO}$ emission in HD 121191, HD 121617, and HD 131488. We detected all three $\mathrm{CO}$ isotopologues in all three targets, except for the $\mathrm{C}^{18} \mathrm{O}$ in $\mathrm{HD} 121191$. Integrating for the velocity ranges where significant line emission can be seen, we calculated zeroth moment maps (Fig. 2 shows these for ${ }^{12} \mathrm{CO}$ and $\left.{ }^{13} \mathrm{CO}\right)$. Next, we determined an aperture which contains all CO flux, measured the integrated line fluxes, and calculated the spectra (Fig. 2). The spectra with higher signal-to-noise ratio show clear Keplerian profiles. For the non-detections, we used a velocity range of $\pm 5 \mathrm{~km} \mathrm{~s}^{-1}$ of the stellar radial velocity and an aperture radius corresponding to 100 au to estimate $3 \sigma \mathrm{CO}$ flux upper limits. Our results are displayed in Tab. 2.

For the three detected sources, we estimated the diskaveraged optical depths of the different CO isotopologues, from the measured line ratios. Assuming identical excitation temperatures, emitting regions, and line widths for all isotopologues the ratio of the ${ }^{12} \mathrm{CO}$ and $\mathrm{C}^{18} \mathrm{O}$ integrated brightness temperatures can be approximated as $\frac{1-e^{-\tau_{12}}}{1-e^{-\tau_{18}}}$ and similar formulae can be used for the ${ }^{13} \mathrm{CO}$ to $\mathrm{C}^{18} \mathrm{O}$ or ${ }^{12} \mathrm{CO}$ to $\mathrm{C}^{13} \mathrm{O}$ line ratios (see, e.g., Lyo et al. 2011).Adopting isotope ratios typical for the local interstellar matter, i.e. $\left[{ }^{12} \mathrm{C}\right] /\left[{ }^{13} \mathrm{C}\right]=77$ and $\left[{ }^{16} \mathrm{O}\right] /\left[{ }^{18} \mathrm{O}\right]=560$ (Wilson \& Rood 1994), we found the ${ }^{12} \mathrm{CO}$ line to be highly optically thick $\left(\tau_{12}>30\right)$ in all three disks. For HD 131488, even the ${ }^{13} \mathrm{CO}$ line is optically thick with an optical depth of $\sim 5.7$, while for HD 121191 and HD 121617 this line was found to be optically thin. Isotope selective photodissociation, and gas phase ion-molecule reactions may lead to different isotopologue ratios in circumstellar disks than in the local interstellar matter (Visser et al. 2009; Miotello et al. 2014). For instance, due to these processes, the $\mathrm{C}^{18} \mathrm{O}$ molecules are expected to be underabundant by up to a factor of 10 (Miotello et al. 2014). It may increase the calculated ${ }^{12} \mathrm{CO}$ and ${ }^{13} \mathrm{CO}$ optical depth, potentially questioning our conclusion of the optically thin ${ }^{13} \mathrm{CO}$ emission in the case of HD 121617.

We used the peak flux values of the optically thick ${ }^{12} \mathrm{CO}$ data to estimate the gas brightness temperature, and obtained $6 \mathrm{~K}, 10 \mathrm{~K}$, and $18 \mathrm{~K}$ for HD 121191 , HD 131488, and HD 121617, respectively. Since the emitting region might be smaller than our beam leading to beam dilution, these values should be considered as lower limits. 
Table 2. Observational data

\begin{tabular}{|c|c|c|c|c|c|c|c|}
\hline Parameters & HD 98363 & HD 109832 & HD 121191 & HD 121617 & HD 131488 & HD 143675 & HD 145880 \\
\hline \multicolumn{8}{|c|}{ Observational and imaging parameters } \\
\hline Obs. date & 2016Mar 22 & 2016 Mar 22 & 2016 May 23 & 2016 May 23 & 2016 May 08 & 2016 May 16 & 2016 May 16 \\
\hline Number of antennas & 37 & 37 & 35 & 35 & 41 & 39 & 39 \\
\hline Baseline lengths (m) & $15.3-460$ & $15.3-460$ & $16.7-640$ & $16.7-640$ & $15.1-640$ & $16.5-640$ & $16.5-640$ \\
\hline \multicolumn{8}{|c|}{ Continuum imaging } \\
\hline Beam size $\left({ }^{\prime \prime}\right)$ & $0 ., 70 \times 0^{\prime \prime} 82$ & $0 . .71 \times 00^{\prime \prime} 86$ & $0^{\prime \prime} 51 \times 0^{\prime \prime} 59$ & $00^{\prime \prime} 49 \times 0^{\prime \prime} 57$ & $00^{\prime \prime} 53 \times 0^{\prime \prime} 55$ & $00^{\prime \prime} 48 \times 0^{\prime \prime} 62$ & $0 .{ }^{\prime \prime} 48 \times 00^{\prime \prime} 64$ \\
\hline Beam PA $\left({ }^{\circ}\right)$ & 32.5 & -6.7 & $-50^{\circ} 3$ & -64.1 & $-34^{\circ} 6$ & -51.9 & $-51^{\circ} 3$ \\
\hline $\operatorname{rms}\left(\mu \mathrm{Jy}_{\text {beam }^{-1}}\right)$ & 26 & 25 & 40 & 42 & 29 & 43 & 43 \\
\hline \multicolumn{8}{|c|}{${ }^{12} \mathrm{CO}(2-1)$ imaging } \\
\hline Beam size $\left({ }^{\prime \prime}\right)$ & $0 . . \prime 71 \times 0 . .84$ & $0 . .^{\prime \prime} 71 \times 00^{\prime \prime} 88$ & $0 .{ }^{\prime \prime} 52 \times 0 .{ }^{\prime \prime} 60$ & $0 . .51 \times 0 .{ }^{\prime \prime} 58$ & $00^{\prime \prime} 54 \times 0^{\prime \prime} 56$ & $00^{\prime \prime} 48 \times 00^{\prime \prime} 62$ & $0 .{ }^{\prime \prime} 48 \times 0 .{ }^{\prime \prime} 65$ \\
\hline Beam PA $\left({ }^{\circ}\right)$ & +29.7 & $-7^{\circ} 1$ & -49.5 & $-63^{\circ} 4$ & $-16^{\circ} 4$ & -50.9 & -49.8 \\
\hline rms (mJy beam ${ }^{-1}$ chan. $^{-1}$ ) & 4.3 & 4.2 & 6.2 & 6.1 & 4.5 & 6.4 & 6.3 \\
\hline \multicolumn{8}{|c|}{${ }^{13} \mathrm{CO}(2-1)$ imaging } \\
\hline Beam size $\left({ }^{\prime \prime}\right)$ & $0 . .73 \times 0^{\prime \prime} .87$ & $0^{\prime \prime} 74 \times 0^{\prime \prime} 91$ & $0 .{ }^{\prime \prime} 54 \times 0^{\prime \prime} 61$ & $0^{\prime \prime} 52 \times 0^{\prime \prime} 60$ & $0^{\prime \prime} 56 \times 0^{\prime \prime} 57$ & $0^{\prime \prime} 50 \times 0.0^{\prime \prime} 65$ & $0 .{ }^{\prime \prime} 50 \times 0.0^{\prime \prime} 67$ \\
\hline Beam PA $\left(^{\circ}\right)$ & +31.6 & -5.5 & -52.2 & -65.0 & -25.7 & -52.9 & -51.5 \\
\hline rms (mJy beam ${ }^{-1}$ chan $^{-1}$ ) & 2.0 & 2.0 & 3.0 & 2.9 & 2.0 & 2.9 & 2.7 \\
\hline \multicolumn{8}{|c|}{$\mathrm{C}^{18} \mathrm{O}(2-1)$ imaging } \\
\hline Beam size $\left({ }^{\prime \prime}\right)$ & $0 .^{\prime \prime} 74 \times 00^{\prime \prime} 88$ & $0 .^{\prime \prime} 75 \times 0^{\prime \prime} 92$ & $0 .{ }^{\prime \prime} 54 \times 0^{\prime \prime} 63$ & $0^{\prime \prime} 53 \times 0^{\prime \prime} 62$ & $0^{\prime \prime} 57 \times 0^{\prime \prime} 58$ & $0^{\prime \prime} 51 \times 0^{\prime \prime} 66$ & $0^{\prime \prime} 51 \times 0^{\prime \prime} 68$ \\
\hline Beam PA $\left({ }^{\circ}\right)$ & $+30^{\circ} 5$ & -5.5 & -49.1 & -63.5 & -21.4 & -52.5 & $-50^{\circ} .7$ \\
\hline rms (mJy beam ${ }^{-1}$ chan $\left.^{-1}\right)$ & 1.6 & 1.6 & 2.3 & 2.1 & 1.6 & 2.3 & 2.3 \\
\hline \multicolumn{8}{|c|}{ Measured continuum flux densities and CO integrated line fluxes } \\
\hline$F_{\nu}$ at $1.33 \mathrm{~mm}(\mathrm{mJy})$ & $0.107 \pm 0.027$ & $<0.105$ & $0.130 \pm 0.029$ & $1.86 \pm 0.29$ & $2.91 \pm 0.31$ & $<0.228$ & $<0.234$ \\
\hline$S_{12 \mathrm{CO}}\left(\mathrm{Jy} \mathrm{km} \mathrm{s}^{-1}\right)$ & $<0.036$ & $<0.036$ & $0.231 \pm 0.044$ & $1.27 \pm 0.13$ & $0.780 \pm 0.084$ & $<0.078$ & $<0.071$ \\
\hline$S_{13 \mathrm{CO}}\left(\mathrm{Jy} \mathrm{km} \mathrm{s}^{-1}\right)$ & $<0.036$ & $<0.034$ & $0.071 \pm 0.022$ & $0.529 \pm 0.065$ & $0.490 \pm 0.054$ & $<0.070$ & $<0.068$ \\
\hline$S_{\mathrm{C} 18_{\mathrm{O}}}\left(\mathrm{Jy} \mathrm{km} \mathrm{s}^{-1}\right)$ & $<0.026$ & $<0.025$ & $<0.050$ & $0.079 \pm 0.023$ & $0.266 \pm 0.033$ & $<0.061$ & $<0.060$ \\
\hline
\end{tabular}

Using an optically thin line of the isotopologue ${ }^{y} \mathrm{C}^{z} \mathrm{O}$, the total ${ }^{12} \mathrm{CO}$ mass can be obtained as:

$$
M_{C O}=4 \pi m d^{2} \frac{S_{u l}}{x_{u} h \nu_{u l} A_{u l}} f^{12} C^{16} O /{ }^{y} C^{z} O,
$$

where $m$ is the mass of the ${ }^{12} \mathrm{CO}$ molecule, $d$ is the distance of the object, $h$ is the Planck constant. $\nu_{u l}$ and $A_{u l}$ are the rest frequency and the Einstein coefficient for the given transition between the $u$ upper and $l$ lower levels, $S_{u l}$ is the observed integrated line flux, $x_{u}$ is the fractional population of the upper level; all these parameters are related to the specific ${ }^{y} \mathrm{C}^{z} \mathrm{O}$ isotopologue. $f^{12} \mathrm{C}^{16} \mathrm{O} /{ }^{y} \mathrm{C}^{z} \mathrm{O}$ is the abun- dance of the ${ }^{12} \mathrm{CO}$ molecule relative to the specific $\mathrm{CO}$ isotopologue (we adopted local interstellar ratios, see above). In our observations we detected the $\mathrm{J}=2-1$ transition of the different $\mathrm{CO}$ isotopologues. By assuming local thermodynamical equilibrium (LTE) and adopting $20 \mathrm{~K}$ for the gas temperature, we determined $x_{2}$ from the Boltzmann equation. We used $\mathrm{C}^{18} \mathrm{O}$ for HD 131488 and ${ }^{13} \mathrm{CO}$ for HD 121191, yielding total CO masses of $8.9 \pm 1.5 \times 10^{-2} \mathrm{M}_{\oplus}$ and $2.7 \pm 0.9 \times 10^{-3} \mathrm{M}_{\oplus}$, respectively. In the case of $\mathrm{HD} 121617$ both the ${ }^{13} \mathrm{CO}$ and $\mathrm{C}^{18} \mathrm{O}$ lines may be optically thin, providing concor- 

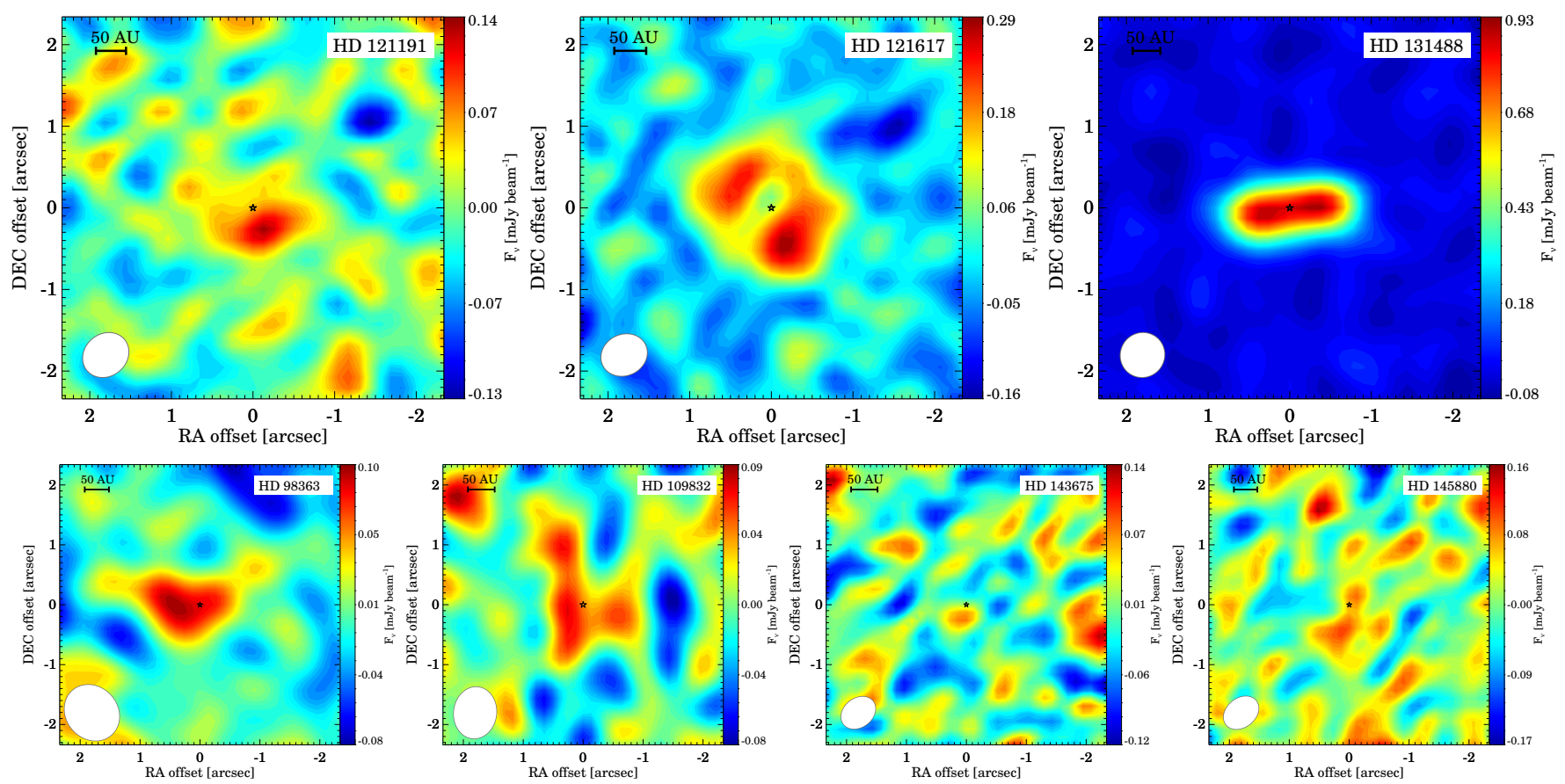

Figure 1. ALMA continuum images at $1.33 \mathrm{~mm}$ of our seven targets. Upper panels show the maps of CO-bearing disks.

dant $\mathrm{CO}$ gas mass estimates of $1.8 \pm 0.3 \times 10^{-2} \mathrm{M}_{\oplus}$ and $2.0 \pm 0.7 \times 10^{-2} \mathrm{M}_{\oplus}$, respectively.

The quoted uncertainties account only for the errors in the measured line fluxes and in the distances. There could be, however, other sources of uncertainty. Similarly to the dust mass estimate, the gas temperature was characterized by a single value instead of a more realistic temperature distribution. Assuming LTE, the fractional population of the upper $\mathrm{J}=2$ level has a maximum at around our adopted gas temperature of $20 \mathrm{~K}$. Taking temperatures lower than $\sim 14 \mathrm{~K}$ or higher than $20 \mathrm{~K}$ we would get lower fractional populations, i.e. higher gas masses. Between gas temperatures of 8 and $48 \mathrm{~K}$, the mass estimate is at most $1.5 \times$ higher the one derived for $20 \mathrm{~K}$.

LTE requires a dense medium where the collisions of $\mathrm{CO}$ with other particles are sufficiently frequent. In lower density environments, however, radiative excitation can dominate over collisional processes. Such a non-LTE situation can lead to weakly populated levels, even at the $\mathrm{J}=2$ rotational level of the $\mathrm{CO}$ molecule, resulting in higher mass estimates. Isotopologue abundance ratios different from the local interstellar values also affect the results. The combination of all these effects may lead to somewhat higher values than our mass estimates in Tab. 1.

Eight out of the other ten disks have been detected in CO lines. For HD 21997, $\beta$ Pic, and HD 131835, the mass estimates were taken from Kóspál et al. (2013),
Matrà et al. (2017a), and Moór et al. in prep., respectively. For the other detected targets (49 Cet, HD 32297, HD 110058, HD 138813, HD 156623) we used their measured ${ }^{12} \mathrm{CO}$ line fluxes (see Tab. 1), and by assuming LTE, we adopted $32 \mathrm{~K}$ for 49 Cet (Hughes et al. 2017), and $20 \mathrm{~K}$ for the other sources as the gas excitation temperature in the $\mathrm{CO}$ mass calculation. Taking into account that the ${ }^{12} \mathrm{CO}$ line could be optically thick in these targets and considering the already mentioned caveats about $\mathrm{CO}$ mass estimates (see above) we considered our results as lower limits (Tab. 1).

\section{DISCOVERY OF THREE NEW GASEOUS DEBRIS DISKS}

HD 131488 - is an A1-type member of the $\sim 16 \mathrm{Myr}-$ old UCL association. Based on its SED, the debris disk has a high fractional luminosity and may contain two belts, the inner one being exceptionally hot (Melis et al. 2013). This system has the highest dust and $\mathrm{CO}$ gas mass among the known gaseous debris disks. The dust emission is elongated in the east-west direction, with a central depression. We fitted the continuum visibilities with the uvmultifit task using a Gaussian ring model (Martí-Vidal et al. 2014). The best-fit parameters are: total flux of $2.9 \pm 0.1 \mathrm{mJy}$, ring diameter of $1^{\prime \prime} 14 \pm 0^{\prime \prime} 04(168 \pm 6 \mathrm{au})$, inclination of $82^{\circ} \pm 3^{\circ}$, position angle of $96 \pm 1^{\circ}$, FWHM of the ring thickness of $00^{\prime \prime} 30 \pm 0 . \prime 08(44 \pm 11 \mathrm{au})$. The center of the model does not show significant offset from the stellar position. The 

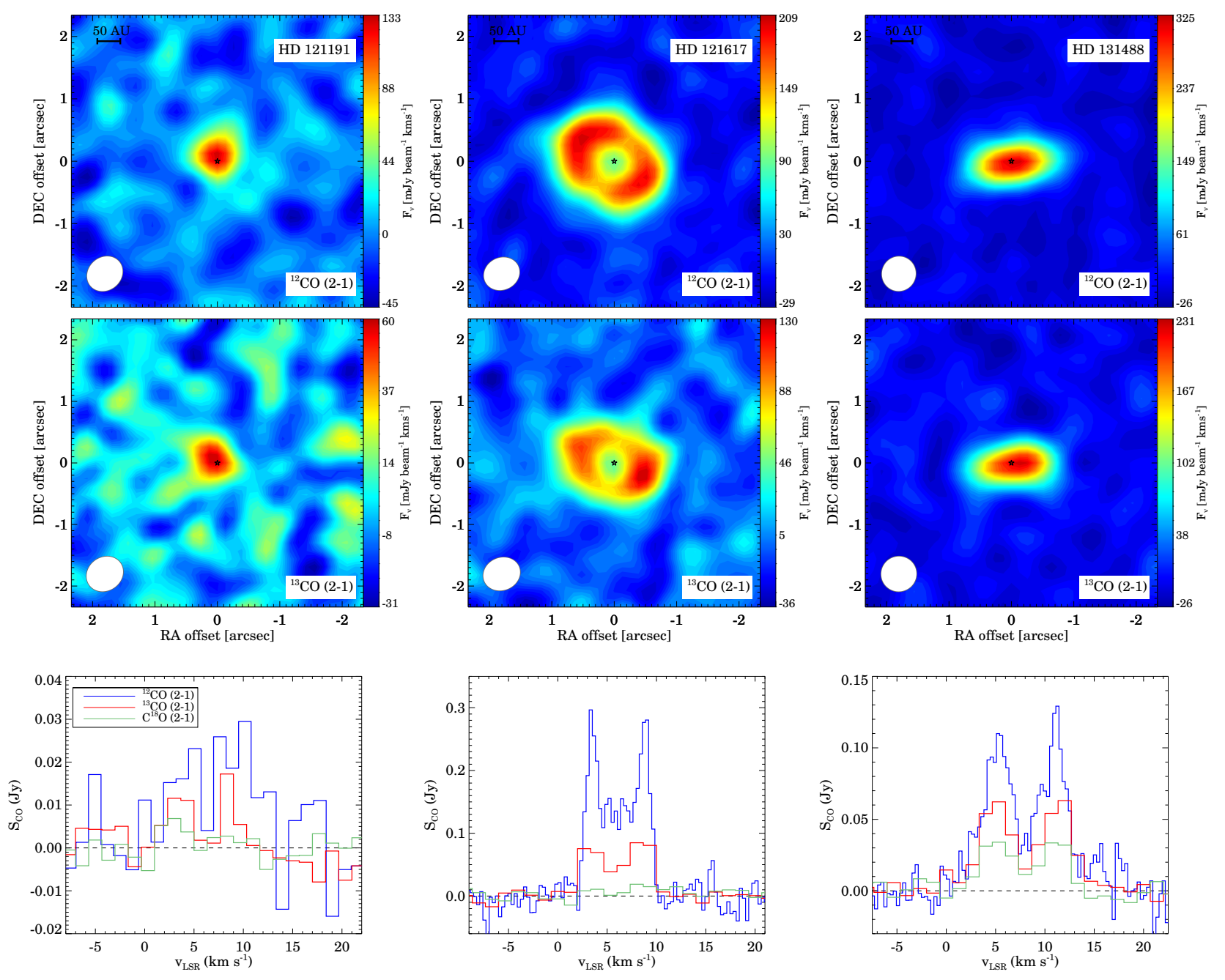

Figure 2. ALMA line data for the three CO-bearing disks. The first and second rows show the ${ }^{12} \mathrm{CO}$ and ${ }^{13} \mathrm{CO}$ zeroth moment maps, respectively, while the third row displays the $\mathrm{CO}$ spectra.

obtained ring may correspond to the cold, outer dust belt inferred from the SED, however, the measured ring radius is $2.7 \pm 0.3$ times larger than the one computed by assuming blackbody grains based on the stellar luminosity and the dust temperature quoted in Tab. 1. Using a sample of 34 spatially resolved debris disks, Pawellek et al. (2014) studied how the ratios of the measured disk radii to the blackbody radii $\left(\Gamma=R_{\text {meas }} / R_{\mathrm{BB}}\right)$ vary as a function of stellar luminosity. By placing our target in their figure $4 \mathrm{~b}$ (where they calculated $\Gamma$ similarly to our analysis), we found that the obtained $\Gamma$ value is higher than any of the other systems with similarly bright host stars, suggesting an overabundance of small grains that are hotter than blackbodies.

HD 121617 - is also an A1-type member of the 16 Myrold UCL association. Its SED shows high fractional luminosity and is consistent with a single ring model with $T_{\text {dust }}=105 \mathrm{~K}$ (Moór et al. 2011). The continuum image shows an inclined ring-like morphology. The uvmultifit procedure yielded the following parameters: total flux of $1.9 \pm 0.2 \mathrm{mJy}$, ring diameter of 1 '. $29 \pm 0$ "' 12 $(165 \pm 16 \mathrm{au})$, inclination of $37^{\circ} \pm 13^{\circ}$, position angle of $43 \pm 19^{\circ}$, FWHM of the ring thickness of $0^{\prime \prime} 44 \pm 0^{\prime \prime} 15$ $(57 \pm 19 \mathrm{au})$. In this case, the ratio of the inferred radius to the blackbody radius is $\Gamma=2.9 \pm 0.4$. This $\Gamma$ value and a stellar luminosity that is higher than that of our previous target, makes HD 121617 an even more prominent outlier in figure 4b of Pawellek et al. (2014), also implying the presence of copious amount of small grains. Remarkably, by analyzing the characteristic grain sizes relative to the theoretical blowout size in 12 spatially resolved young debris disks, Lieman-Sifry et al. (2016) found that the CO-bearing systems in the sample had grain sizes on the small end of the distribution, and two of them had grain sizes smaller than the blowout size. 
HD 121191. - The position and space motion of this A5-type star can be consistent with either the UCL ( $\sim 16 \mathrm{Myr})$ or LCC ( 15 Myr) sub-groups of the ScoCen association (Melis et al. 2013). Based on its SED, it has a high fractional luminosity and it may also have two belts, the inner one being very hot and bright (Melis et al. 2013). The weak $1.3 \mathrm{~mm}$ continuum emission from this target is unresolved, and its peak is offset from the stellar position toward the SW $\left(\Delta \mathrm{RA}=-0 .{ }^{\prime \prime} 16 \pm 0 . \prime 05 ; \Delta \mathrm{Dec}=-0 . \prime 17 \pm 0 .{ }^{\prime} 05\right)$. This may be an axisymmetric ring whose parts remain below the noise level, or may be a ring with highly asymmetric brightness distribution, although contamination by a background source cannot be excluded either. Both ${ }^{12} \mathrm{CO}$ and ${ }^{13} \mathrm{CO}$ were firmly detected as compact sources centered on the stellar position. This disk has a lower dust mass than the previous two targets.

\section{MOLECULAR GAS IN BRIGHT DEBRIS DISKS}

Until recently, only $\beta$ Pic and 49 Cet were known as CO-bearing debris disks (Vidal-Madjar et al. 1994; Zuckerman et al. 1995; Roberge et al. 2000). Later, more such objects were discovered, and the first statistical studies could be done (Lieman-Sifry et al. 2016; Greaves et al. 2016; Péricaud et al. 2017). Our present list partly incorporates these earlier samples, and extends them to a full list of 17 dust-rich debris disks in the Solar neighborhood (Sec. 2). In Fig. 3a we display the ${ }^{12} \mathrm{CO} 2-1$ (or ${ }^{12} \mathrm{CO} 3-2$ for HR 4796) line luminosities (or upper limits), as a function of fractional luminosity for our sample. Line luminosities of detected CObearing disks span almost two orders of magnitude, the brightest disks have luminosities comparable to those of fainter Herbig Ae and T Tauri disks (Ansdell et al. 2016; Péricaud et al. 2017). Since the sensitivity of the HR 4796 observation is nearly two orders of magnitude worse than that of the other measurements, we discard this object from the following analysis, reducing the size of our statistical sample to 16 . For the other objects, adopting the highest upper limit (source no. 7) in Fig. 3a, we derived a detectability threshold of $\sim 1.4 \times 10^{4} \mathrm{Jy} \mathrm{km} \mathrm{s}^{-1} \mathrm{pc}^{2}$ for the ${ }^{12} \mathrm{CO} 2-1$ line luminosity. With our three new discoveries, we found 11 disks in this sample that harbor $\mathrm{CO}$ gas, resulting in a very high detection rate of $68.8_{-13.1}^{+8.9} \%$. Because of the small sample, we computed the uncertainties (corresponding to $68 \%$ confidence interval) using the binomial distribution approach proposed by Burgasser et al. (2003). Our result indicates that the presence of $\mathrm{CO}$ gas in dust-rich debris disks around young A-type stars is likely more the rule than the exception. The obtained incidence rate of $11 / 16$ is valid above our detectability threshold for the ${ }^{12} \mathrm{CO} \mathrm{J}=2-1$ line luminosities. Nevertheless we cannot rule out that all of our targets harbor $\mathrm{CO}$ gas at some level. Remarkably, as Fig. 3a shows, apart from HR 4796, all disks with $L_{\text {disk }} / L_{\text {bol }}>2 \times 10^{-3}$, contain detectable levels of $\mathrm{CO}$ gas.

Dividing our list into two subsamples that contain stars having lower and higher luminosity than $10 \mathrm{~L}_{\odot}$, we get $\mathrm{CO}$ detection rates of $62.5_{-17.9}^{+12.8 \%}(5 / 8)$ and $75.0_{-19.3}^{+9.1} \%(6 / 8)$, respectively. This implies that there is no evidence in support of a correlation with stellar luminosity within the A-type sample.

To compare with debris disks around later type stars, we collected previous ALMA results for those young (1050 Myr) debris disks that encircle F- and G-type stars, but otherwise fulfill our original selection criteria (Sec. 2) and their ALMA measurements achieved the same detection threshold $\left(\sim 1.4 \times 10^{4} \mathrm{Jy} \mathrm{km} \mathrm{s}^{-1} \mathrm{pc}^{2}\right)$ as that of our A-type sample. We found 16 such systems: 14 belonging to the Sco-Cen association (Lieman-Sifry et al. 2016), and two young moving group members, HD 61005 and HD 181327 (Olofsson et al. 2016; Marino et al. 2016). From the FG stars, two objects were found to harbor CO gas (HD 181327 and HD 146897). However, with its ${ }^{12} \mathrm{CO} 2-1$ line luminosity of $\sim 10^{3} \mathrm{Jy}_{\mathrm{km} \mathrm{s}}{ }^{-1} \mathrm{pc}^{2}$ (based on Marino et al. 2016), HD 181327 is at least an order of magnitude fainter than any of the other known gaseous disks from the studied samples. Its detection was only possible because of its proximity and the long exposure time. Such a faint CO disk would not be detectable at any objects in the A-type sample, therefore this source was discarded from the following analysis, yielding a detection rate of $6.7_{-2.2}^{+12.5} \%$ for disks around young FG-type stars. By applying a Fisher's exact test to compare the occurrence of CO-bearing disks in the two samples, we obtained a $p$ value of $6.4 \times 10^{-4}$, implying a statistically significant difference between dustrich debris disks around young A-type and FG-type stars above our detectability threshold. This suggests that either the incidence of $\mathrm{CO}$ gas is truly lower around young FG-type star than in A-type systems, or the CO line luminosities are systematically lower. The reason for the latter may be related to lower $\mathrm{CO}$ gas content and/or weaker population of the $\mathrm{J}=2$ rotational level due to less efficient excitation (in optically thin case), or systematically lower CO temperatures and/or disk sizes (in optically thick case).

In Fig. 3bc, we plotted $M_{\text {dust }}$ and $M_{\mathrm{CO}}$ versus age for CO-bearing debris disks. To study the transition between primordial and debris disks we also plotted some Herbig $\mathrm{Ae} / \mathrm{Fe}$ systems with $\mathrm{CO}$ isotopologue observations from the literature. There is a pronounced drop in dust mass from primordial to debris disks (see also, 

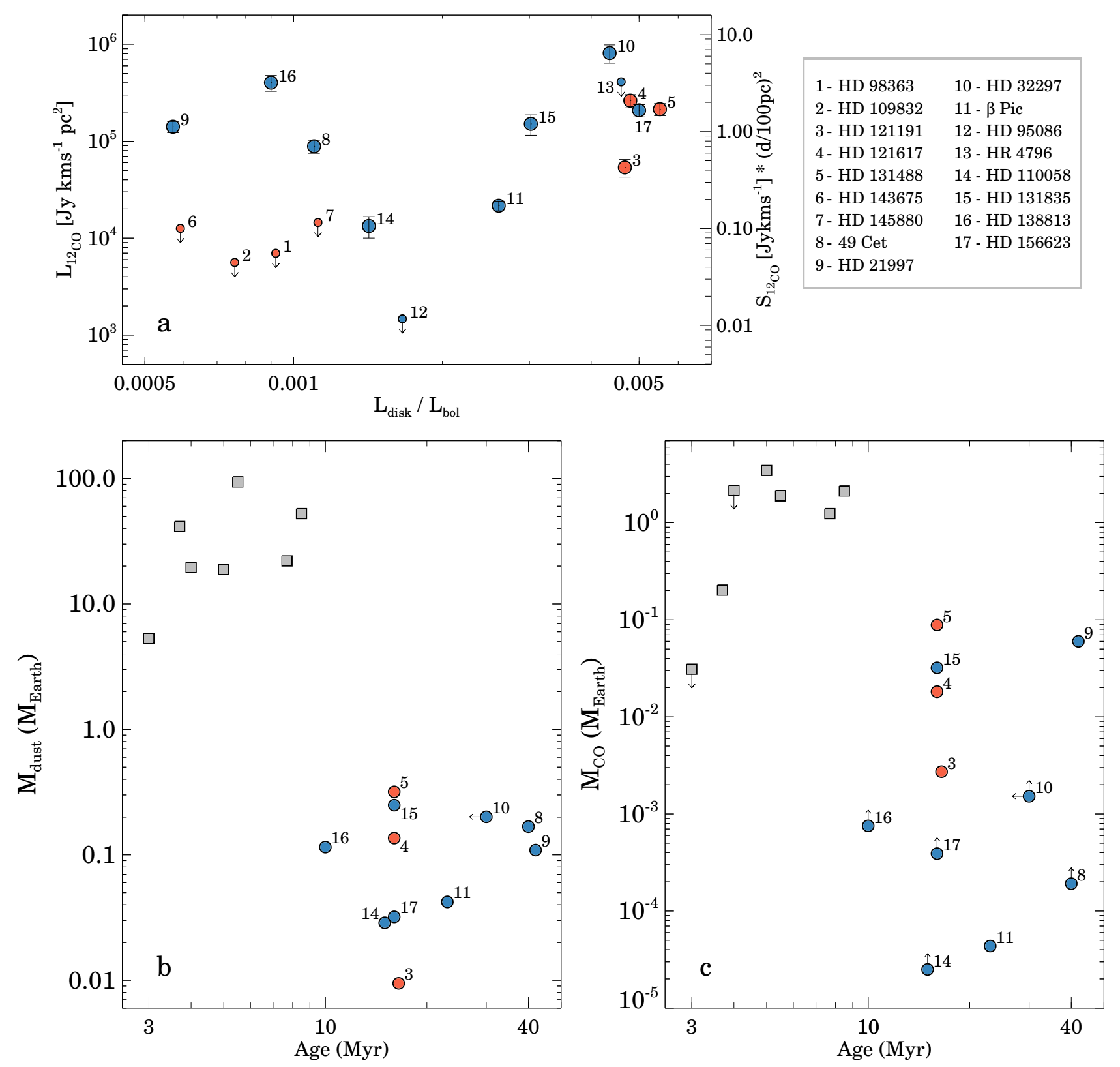

Figure 3. a) ${ }^{12} \mathrm{CO} 2-1$ (3-2 for HR 4796) line luminosity for the selected 17 bright debris disks as a function of fractional luminosity. For the sake of compatibility with previous works, integrated line fluxes normalized to $100 \mathrm{pc}$ are also drawn. Our current seven targets are marked by red symbols. Sources with no CO detection are displayed with smaller symbols. b) Dust masses of circumstellar disks derived from observed (sub)millimetre fluxes towards disks around some selected Herbig stars (gray squares), and CO-bearing debris disks. For consistency, millimeter fluxes of Herbig disks (taken from Raman et al. 2006; Isella et al. 2007; Chapillon et al. 2008; Öberg et al. 2010; Ansdell et al. 2016; Péricaud et al. 2017) were converted to dust masses by adopting an opacity of $\kappa_{\nu}=2.3\left(\frac{\nu}{230 \mathrm{GHz}}\right)^{0.7}$ and a dust temperature of $50 \mathrm{~K}$. c) CO gas masses derived from optically thin ${ }^{12} \mathrm{CO}$ or $\mathrm{CO}$ isotopologue $\left({ }^{13} \mathrm{CO}, \mathrm{C}^{17} \mathrm{O}\right.$ or $\left.\mathrm{C}^{18} \mathrm{O}\right)$ line observations for the same objects. To compute CO masses for Herbig sample, we assumed LTE and a gas excitation temperature of $50 \mathrm{~K}$. CO isotopologue data are from Rosenfeld et al. (2013); Guilloteau et al. (2013, 2016); Ansdell et al. (2016); Fedele et al. (2017). 
Roccatagliata et al. 2009; Panić et al. 2013). In CO gas mass, however, debris disks show a significantly larger spread. Four gaseous debris disks - HD 121617 and HD 131488 from our current sample, as well as HD 21997 and HD 131835 - harbor comparable amounts of CO to that of the two least massive Herbig disks in the figure. For these disks, our ALMA CO isotopologue observations revealed $30-250 \times$ more $\mathrm{CO}$ gas than that calculated from ${ }^{12} \mathrm{CO}$ measurements (using an optically thin assumption), highlighting the importance of less abundant molecules in reliable mass estimates.

While the gas in the disk of $\beta$ Pic, and around the two F-type hosts is secondary (Kral et al. 2017; Marino et al. 2016; Matrà et al. 2017a), the presence of significantly larger amounts of CO gas at HD 21997 and HD 131835 indicates that these systems may have hybrid disks, where the gas is leftover from the primordial phase (Kóspál et al. 2013; Kral et al. 2017, Moór et al. in prep.). The likely existence of hybrid disks would be a strong indication that in some systems the evolution of dust and gas, at least in the outer disk, is decoupled. By exhibiting CO masses similar to that of HD 21997 and HD 131835, the three newly discovered CO-bearing disks, particularly HD 121617 and HD 131488, could be prime hybrid disk candidates. The origin of gas in these systems, along with their detailed morphological analysis, will be the topic of a next paper. Four gaseous systems, 49 Cet, HD 32297, HD 138813, and HD 156623 have similar ${ }^{12} \mathrm{CO} 2-1$ line luminosity to the above subsample, therefore their current $\mathrm{CO}$ mass estimates should be considered as lower limits. The origin of gas in these systems is not known. Further observations at
$\mathrm{CO}$ isotopologue lines might reveal that some of them may exhibit hybrid nature as well. If the disks with the highest ${ }^{12} \mathrm{CO}$ line luminosities in Fig. 3a indeed turn out to be hybrid disks, it would indicate a strong link between the hybrid disk phenomenon and young A-type stars. If this link is exclusive, then it could help to understand why we detect a lower number of gaseous debris disks around FG-type stars above our current detectability threshold.

We acknowledge the referee for insightful comments that have improved the paper. We appreciate Ivan Martí-Vidal for helpful advices on the uvmultifit tool. This paper makes use of the following ALMA data: ADS/JAO.ALMA\#2015.1.01243.S. ALMA is a partnership of ESO (representing its member states), NSF (USA) and NINS (Japan), together with NRC (Canada) and NSC and ASIAA (Taiwan) and KASI (Republic of Korea), in cooperation with the Republic of Chile. The Joint ALMA Observatory is operated by ESO, AUI/NRAO and NAOJ. This work was supported by the Momentum grant of the MTA CSFK Lendület Disk Research Group and Hungarian OTKA grant K119993. A.M.H. gratefully acknowledges support from NSF grant AST-1412647. Este trabajo conta con el apoyo de CONICYT Programa de Astronomia Fondo ALMA-CONICYT, cargo proyectos numeros: 31140024 y $31 \mathrm{AS} 002$.

\section{Facilities: ALMA}

\section{REFERENCES}

Alexander, R., Pascucci, I., Andrews, S., Armitage, P., \& Cieza, L. 2014, Protostars and Planets VI, ed. H.

Beuther et al. (Tucson, AZ: Univ. Arizona Press), 475

Andrews, S. M., Rosenfeld, K. A., Kraus, A. L., \& Wilner, D. J. 2013, ApJ, 771, 129

Ansdell, M., Williams, J. P., van der Marel, N., et al. 2016, ApJ, 828, 46

Ballering, N. P., Rieke, G. H., Su, K. Y. L., \& Montiel, E. 2013, ApJ, 775, 55

Barrado y Navascués, D., Stauffer, J. R., Song, I., \& Caillault, J.-P. 1999, ApJL, 520, L123

Bell, C. P. M., Mamajek, E. E., \& Naylor, T. 2015, MNRAS, 454, 593

Burgasser, A. J., Kirkpatrick, J. D., Reid, I. N., et al. 2003, ApJ, 586, 512
Chapillon, E., Guilloteau, S., Dutrey, A., \& Piétu, V. 2008, A\&A, 488, 565

Chen, C. H., Mittal, T., Kuchner, M., et al. 2014, ApJS, 211,25

Dent, W. R. F., Wyatt, M. C., Roberge, A., et al. 2014, Science, 343, 1490

Fedele, D., Carney, M., Hogerheijde, M. R., et al. 2017, A\&A, 600, A72

Greaves, J. S., Holland, W. S., Matthews, B. C., et al. 2016, MNRAS, 461, 3910

Guilloteau, S., Di Folco, E., Dutrey, A., et al. 2013, A\&A, 549, A92

Guilloteau, S., Reboussin, L., Dutrey, A., et al. 2016, A\&A, 592, A124

Hales, A. S., De Gregorio-Monsalvo, I., Montesinos, B., et al. 2014, AJ, 148, 47 
Hoogerwerf, R. 2000, MNRAS, 313, 43

Hughes, A. M., Wilner, D. J., Kamp, I., \& Hogerheijde, M. R. 2008, ApJ, 681, 626

Hughes, A. M., Lieman-Sifry, J., Flaherty, K. M., et al. 2017, ApJ, 839, 86

Isella, A., Testi, L., Natta, A., et al. 2007, A\&A, 469, 213

Kalas, P. 2005, ApJL, 635, L169

Kóspál, Á., Moór, A., Juhász, A., et al. 2013, ApJ, 776, 77

Kral, Q., Matrà, L., Wyatt, M. C., \& Kennedy, G. M. 2017, MNRAS, 469, 521

Lieman-Sifry, J., Hughes, A. M., Carpenter, J. M., et al. 2016, ApJ, 828, 25

Lindegren, L., Lammers, U., Bastian, U., et al. 2016, A\&A, 595, A4

Lyo, A.-R., Ohashi, N., Qi, C., Wilner, D. J., \& Su, Y.-N. 2011, AJ, 142, 151

Mamajek, E. E., \& Bell, C. P. M. 2014, MNRAS, 445, 2169

Marino, S., Matrà, L., Stark, C., et al. 2016, MNRAS, 460, 2933

Marino, S., Wyatt, M. C., Panić, O., et al. 2017, MNRAS, 465, 2595

Martí-Vidal, I., Vlemmings, W. H. T., Muller, S., \& Casey, S. 2014, A\&A, 563, A136

Matrà, L., Dent, W. R. F., Wyatt, M. C., et al. 2017, MNRAS, 464, 1415

Matrà, L., MacGregor, M. A., Kalas, P., et al. 2017, arXiv:1705.05868

Matthews, B. C., Krivov, A. V., Wyatt, M. C., Bryden, G., \& Eiroa, C. 2014, Protostars and Planets VI, 521

McMullin, J. P., Waters, B., Schiebel, D., Young, W., \& Golap, K. 2007, in Astronomical Society of the Pacific Conference Series, Vol. 376, Astronomical Data Analysis Software and Systems XVI, ed. R. A. Shaw, F. Hill, \& D. J. Bell, 127

Meeus, G., Montesinos, B., Mendigutía, I., et al. 2012, A\&A, 544, A78

Melis, C., Zuckerman, B., Rhee, J. H., et al. 2013, ApJ, 778,12

Miotello, A., Bruderer, S., \& van Dishoeck, E. F. 2014, A\&A, 572, A96

Miyake, K., \& Nakagawa, Y. 1993, Icarus, 106, 20

Moór, A., Ábrahám, P., Derekas, A., et al. 2006, ApJ, 644, 525

Moór, A., Ábrahám, P., Juhász, A., et al. 2011, ApJL, 740, L7

Moór, A., Henning, T., Juhász, A., et al. 2015a, ApJ, 814, 42
Moór, A., Kóspál, Á., Ábrahám, P., et al. 2015b, MNRAS, 447,577

Öberg, K. I., Qi, C., Fogel, J. K. J., et al. 2010, ApJ, 720, 480

Olofsson, J., Samland, M., Avenhaus, H., et al. 2016, A\&A, 591, A108

Ossenkopf, V., \& Henning, T. 1994, A\&A, 291, 943

Panić, O., Holland, W. S., Wyatt, M. C., et al. 2013, MNRAS, 435, 1037

Pawellek, N., Krivov, A. V., Marshall, J. P., et al. 2014, ApJ, 792, 65

Pecaut, M. J., \& Mamajek, E. E. 2016, MNRAS, 461, 794

Péricaud, J., Di Folco, E., Dutrey, A., Guilloteau, S., \& Piétu, V. 2017, A\&A, 600, A62

Raman, A., Lisanti, M., Wilner, D. J., Qi, C., \& Hogerheijde, M. 2006, AJ, 131, 2290

Rhee, J. H., Song, I., Zuckerman, B., \& McElwain, M. 2007, ApJ, 660, 1556

Riviere-Marichalar, P., Barrado, D., Montesinos, B., et al. 2014, A\&A, 565, A68

Roberge, A., Feldman, P. D., Lagrange, A. M., et al. 2000, ApJ, 538, 904

Roccatagliata, V., Henning, T., Wolf, S., et al. 2009, A\&A, 497, 409

Rodigas, T. J., Debes, J. H., Hinz, P. M., et al. 2014, ApJ, 783, 21

Rosenfeld, K. A., Andrews, S. M., Hughes, A. M., Wilner, D. J., \& Qi, C. 2013, ApJ, 774, 16

Sheret, I., Dent, W. R. F., \& Wyatt, M. C. 2004, MNRAS, 348,1282

Torres, C. A. O., Quast, G. R., Melo, C. H. F., \& Sterzik, M. F. 2008, Young Nearby Loose Associations, ed. B. Reipurth, 757

van Leeuwen, F. 2007, A\&A, 474, 653

Vican, L., Schneider, A., Bryden, G., et al. 2016, ApJ, 833, 263

Vidal-Madjar, A., Lagrange-Henri, A.-M., Feldman, P. D., et al. 1994, A\&A, 290, 245

Visser, R., van Dishoeck, E. F., \& Black, J. H. 2009, A\&A, 503,323

Webb, R. A., Zuckerman, B., Platais, I., et al. 1999, ApJL, $512, \mathrm{~L} 63$

Williams, J. P., \& Andrews, S. M. 2006, ApJ, 653, 1480

Wilson, T. L., \& Rood, R. 1994, ARA\&A, 32, 191

Wyatt, M. C. 2008, ARA\&A, 46, 339

Wyatt, M. C., Panić, O., Kennedy, G. M., \& Matrà, L. 2015, Ap\&SS, 357, 103

de Zeeuw, P. T., Hoogerwerf, R., de Bruijne, J. H. J., Brown, A. G. A., \& Blaauw, A. 1999, AJ, 117, 354

Zuckerman, B., Forveille, T., \& Kastner, J. H. 1995, Nature, 373, 494

Zuckerman, B., \& Song, I. 2012, ApJ, 758, 77 